\title{
On the relationship between auroral and nebular oxygen line intensities in spectra of $\mathrm{H} \|$ regions
}

\author{
L. S. Pilyugin \\ Main Astronomical Observatory of National Academy of Sciences of Ukraine, 27 Zabolotnogo str., 03680 Kiev, Ukraine \\ e-mail: pilyugin@mao.kiev.ua \\ Received 14 Marth 2005 / Accepted 10 April 2005

\begin{abstract}
We investigate relationships between observed auroral and nebular oxygen line fluxes in spectra of $\mathrm{H}$ II regions. We find a relation that is metallicity-dependent at low metallicities, but becomes independent of metallicity (within the uncertainties auroral line from strong oxygen line measurements only. This solves the problem of the electron temperature (and, consequently, abundance) determination in high-metallicity H II regions. The ff-relation confirms the basic idea of the "empirical" method, proposed by Pagel et al. (1979, MNRAS, 189, 95) a quarter of a century ago, that the oxygen abundance in H II region can be esimated from the strong oxygen lines measurements only.
\end{abstract} \\ of the available data) above $12+\log \mathrm{O} / \mathrm{H} \sim 8.25$, i.e. there is one-to-one correspondence (the ff-relation) between auroral and \\ nebular oxygen line fluxes in spectra of high-metallicity H II regions. The ff-relation allows one to estimate the flux in the
}

Key words. galaxies: ISM - ISM: H II region: abundances

\section{Introduction}

Accurate abundances in $\mathrm{H}$ II regions can be derived via the classic $T_{\mathrm{e}}$-method, $T_{\mathrm{e}}$ being the electron temperature of the $\mathrm{H}$ II region. Measurements of faint auroral lines, such as [O III] $\lambda 4363$, are necessary to determine $T_{\mathrm{e}}$. Unfortunately, the auroral lines drop below detectability in the spectra of high-metallicity H II regions. The electron temperature $T_{\mathrm{e}}$ is an indicator of the physical conditions in H II regions. Pilyugin (2000, 2001a,b, 2003) advocated that the physical conditions in an $\mathrm{H}$ II region can be estimated via the excitation parameter $P$, i.e. using the strong oxygen nebular lines only. If this is the case then a relationship between oxygen auroral and nebular line fluxes in spectra of $\mathrm{H}$ II regions must exist. This problem is examined empirically here.

We will be using the following notations throughout the paper: $R_{2}=I_{[\mathrm{OII}] \lambda 3727+\lambda 3729} / I_{\mathrm{H} \beta}, R_{3}=I_{[\mathrm{OIII}] \lambda 4959+\lambda 5007} / I_{\mathrm{H} \beta}$, $R=I_{[\mathrm{OIII}] \lambda 4363} / I_{\mathrm{H} \beta}, R_{23}=R_{2}+R_{3}$. With these definitions, the excitation parameter $P$ can be expressed as: $P=R_{3} /\left(R_{2}+R_{3}\right)$.

\section{Observational data: Line fluxes}

We need to select a sample of H II regions which have oxygen line fluxes (including the auroral line [O III] 14363 ) measured with high precision. The sample of low-metallicity H II regions in blue compact dwarf galaxies observed by Izotov with colleagues (Fricke et al. 2001; Guseva et al. 2001, 2003a,b; Izotov \& Thuan 1998, 2004; Izotov et al. 1994, 1997, 2001, 2004; Noeske et al. 2000; Thuan et al. 1995, 1999) meets that precision criterion. It constitutes one of the largest and most homogeneous data sets now available, being obtained and reduced in the same way. However, this sample contains only a few high-metallicity H II regions. Additional recent measurements of the oxygen line fluxes in high-metallicity $(12+\log \mathrm{O} / \mathrm{H}>8.25) \mathrm{H}$ II regions were taken from Caplan et al. (2000); Esteban et al. (2004, 2005); García-Rojas et al. (2004); Kennicutt et al. (2003); Lee et al. (2003); Oey \& Shields (2000); Oey et al. (2000); Peimbert (2003); Tsamis et al. (2003); Vermeij et al. (2002); Vílchez \& Iglesias-Páramo (2003). The spectroscopic data so assembled form the basis of the present study.

\section{Relationship}

The oxygen abundances in $\mathrm{H}$ II regions from our sample are in the range from $12+\log \mathrm{O} / \mathrm{H} \sim 7.2$ to $\sim 8.6$. The auroral line flux $R$ is shown as a function of the total flux in strong lines $R_{23}$ in Fig. 1 (top panel). The plus signs are $\mathrm{H}$ II regions with $7.4<12+\log \mathrm{O} / \mathrm{H}<7.6$. The crosses are those with $7.8<12+\log \mathrm{O} / \mathrm{H}<8.0$. The circles (filled + open) are those with $12+\log \mathrm{O} / \mathrm{H}>8.25$. Inspection of Fig. 1 shows that the flux in the auroral line $R$ is linked to the total flux in the strong nebular lines $R_{23}$ through a relation of the type

$\log R=a+b \times \log R_{23}$.

Equation (1) will be referred to hereinafter as the flux-flux or ff-relation. In the general case, the coefficient $a$ (and may be also $b$ ) in the ff-relation is a function of the metallicity.

The top panel of Fig. 1 shows that all the $\mathrm{H}$ II regions with $12+\log \mathrm{O} / \mathrm{H}>8.25$ lie along a single line, i.e. the coefficient $a$ 

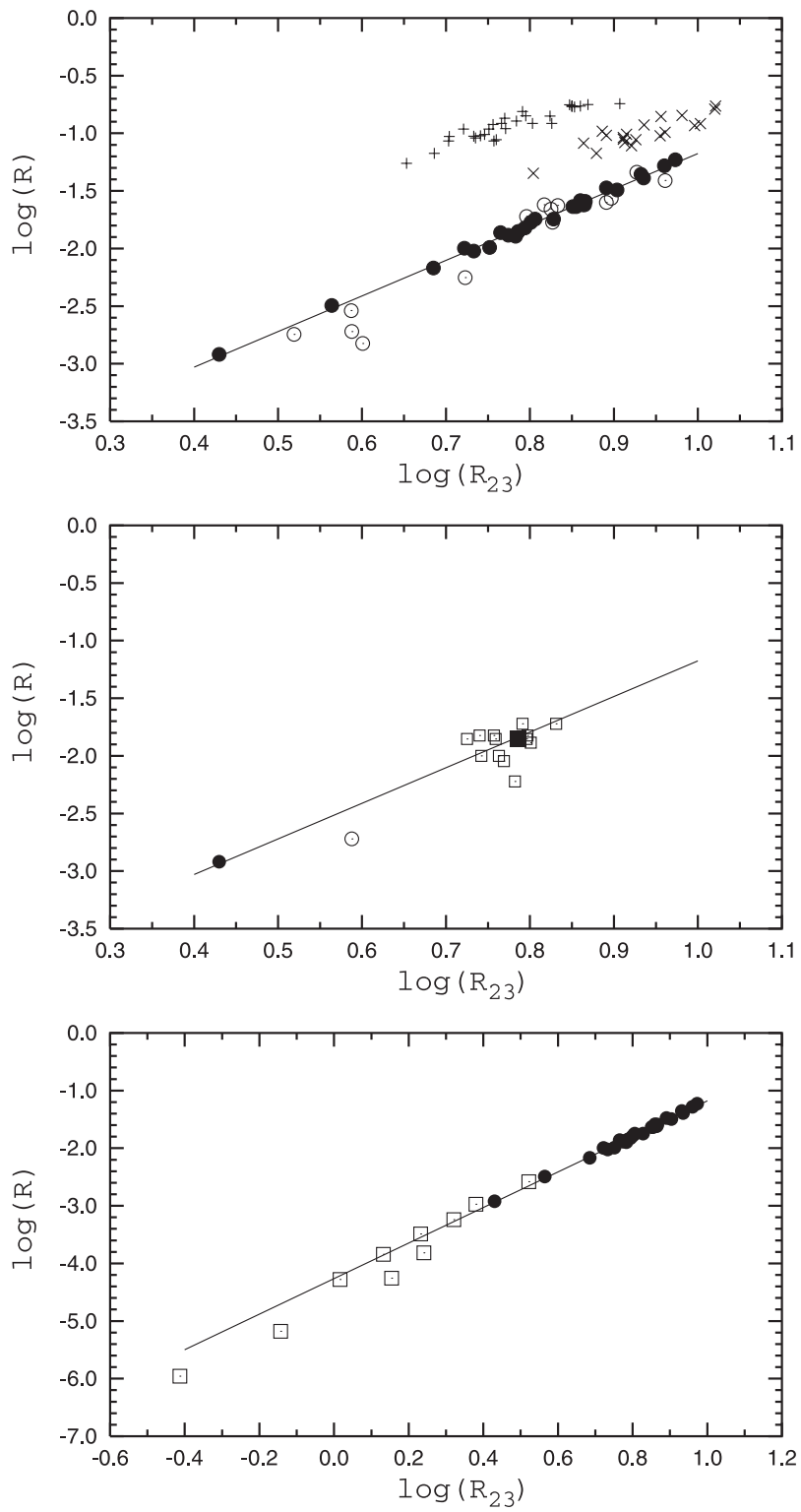

Fig. 1. Top panel. Flux in the oxygen auroral line $R$ as a function of the total flux in strong nebular lines $R_{23}$. The crosses are $\mathrm{H}$ II regions with $7.8<12+\log \mathrm{O} / \mathrm{H}<8.0$. The plus signs are those with $7.4<$ $12+\log \mathrm{O} / \mathrm{H}<7.6$. The circles (open + filled) are $\mathrm{H}$ II regions with $12+\log \mathrm{O} / \mathrm{H}>8.25$, the filled circles are points used for determination of the ff-relation (the solid line). Middle panel. The open squares are measurements for individual areas of the H II region DEM 323 in the Large Magellanic Cloud, the filled square is the integrated data (Oey $\&$ Shields 2000; Oey et al. 2000). The filled circle is the measurement for the area of the H II region M 16 in the Milky Way Galaxy from Caplan et al. (2000), the open circle is that for the same H II region from Esteban et al. (2005). The line is the same as in the top panel. Bottom panel. The open squares are faint $\mathrm{H}$ II regions from Bresolin et al. (2004). The values of $R$ for those $\mathrm{H}$ II regions are estimated from the electron temperatures (see text). The filled circles and the line are the same as in the top panel.

becomes metallicity-independent at high metallicities (or this dependence becomes very weak and is masked by errors in the flux measurements.) However, the scatter in the $R-R_{23}$ diagram for high-metallicity objects is appreciable even for the recent measurements from the publications cited above. Therefore, the ff-relation is derived by using an iteration procedure. In a first step, the relation is determined from all data through the least squares method. Then, the point with largest deviation $(\Delta \log R)_{\max }$ is rejected, and a new relation is derived. The iteration procedure is stopped at the value of $(\Delta \log R)_{\max }= \pm 0.05$ (the filled circles in the top panel of Fig. 1). The following values of the coefficients were obtained: $a=-4.264 \pm 0.038$ and $b=3.087 \pm 0.046$. The ff-relation is shown in Fig. 1 by the line.

Is the scatter in the $R-R_{23}$ diagram real? If this is the case then it is necessary to establish the origin of the scatter, i.e. to establish a "third" parameter which is responsible for the scatter in the ff-relation. Small deviations of positions of H II regions from the ff-relation can be naturally explained by the uncertainties in the flux measurements. It should be emphasized that the largest contribution to the deviation $\Delta(\log R)$ of the measured value of $\log R$ from the line does not necessarily come from errors in the measurement of $R$. In fact, a large error in the measurement (and/or in the dereddening) of the weak auroral line (say, $40 \%$ ) and much smaller (10\%) error of the strong nebular lines result in the same value of the discrepany $\Delta(\log R) \sim 0.15$ dex, since $R \sim R_{23}^{3.087}$. Examination of the top panel of Fig. 1 shows that three Galactic H II regions (M 16, M 20 and S 311 from the list of Esteban et al. (2005)) have large deviations from the line, of order 0.2 dex. It does not seem possible to explain this from errors in the measurements.

There are other reasons why an $\mathrm{H}$ II region may give measurements lying off the line. It has been noted (Pilyugin 2001a) that a combination of the nebular line fluxes (the excitation parameter $\mathrm{P}$ ) is a good indicator of the physical conditions in an $\mathrm{H}$ II region if two conditions are satisfied; $i$ ) the measured fluxes reflect their relative contributions to the radiation of the whole nebula; $i$ ) the $\mathrm{H}$ II region is ionization-bounded. It can be suggested from general considerations that the ff-relation also depends on these two conditions being satisfied. This suggests that the deviation from the ff-relation can occur if the line fluxes measured within the slit do not reflect their relative contributions to the radiation of the nebula as a whole. This would happen, for example, if the relative fractions of the [O II] and [O III] zones in the slit differ significantly from those of the entire nebula. This effect would be more important for extended $\mathrm{H}$ II regions or $\mathrm{H}$ II regions with multiple emission peaks (due, e.g., to spatially distributed separate ionization sources) when only a small fraction of such an H II region is within the slit. This suggestion can be tested in the following way. The middle panel of Fig. 1 shows the measurements for multiple positions in the H II region DEM 323 in the Large Magellanic Cloud (the open squares) and the integrated data (the filled square) from Oey \& Shields (2000); Oey et al. (2000). The integrated data are in agreement with the ff-relation while those for some individual areas show significant deviations. Comparison between integrated fluxes and those for individual areas shows that the largest deviation occurs when the relative contribution of $R_{2}$ flux to $R_{23}$ is significantly higher than in the integrated spectrum.

There are two recent measurements of the oxygen line fluxes in the Galactic H II region M 16 (Caplan et al. 2000; 

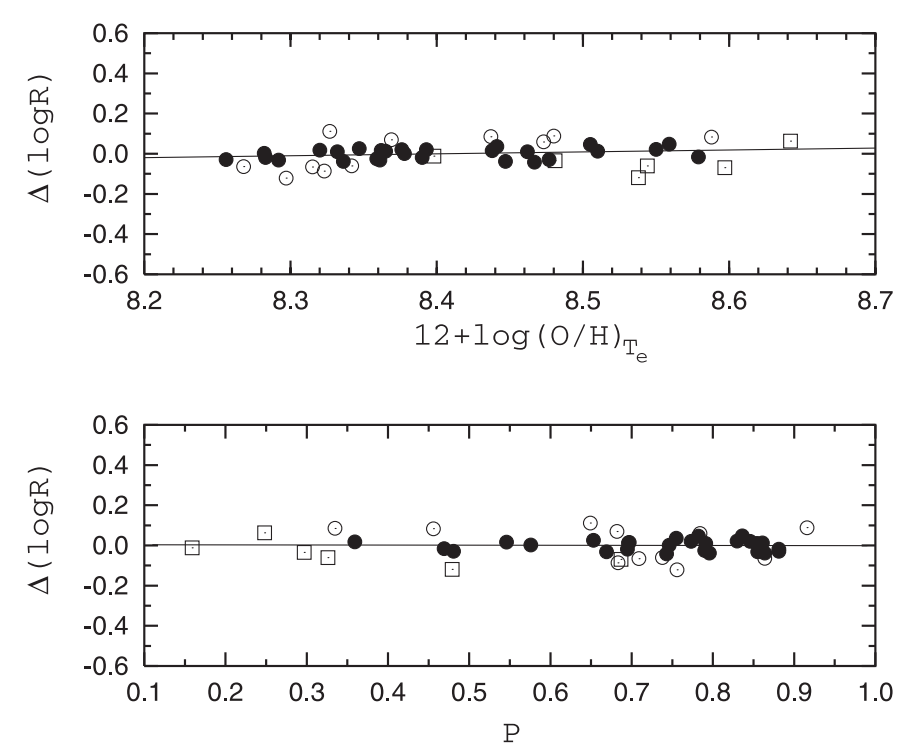

Fig. 2. The residuals $\Delta(\log R)$ from the ff-relation as a function of oxygen abundance (top panel) and of excitation parameter P (bottom panel). The filled circles are points used for the determination of the ff-relation, the lines are best fits to those data. The open circles are other $\mathrm{H}$ II regions with measured $R$. The open squares are $\mathrm{H}$ II regions from Bresolin et al. (2004) with $R$ estimated.

Esteban et al. 2005). Its position based on the data of Caplan et al. (2000) (the filled circle in the middle panel of Fig. 1) is very close to the ff-relation, whereas those of Esteban et al. (2005) (shown by the open circle) show a large deviation. Again, the relative contribution of the $R_{2}$ flux in the spectrum is significantly higher in the case of large deviation from the ff-relation than that in the case of no (or small) deviation.

The ff-relation was derived on the basis of bright $\mathrm{H}$ II regions. Bresolin et al. (2004) have recently detected the auroral line [S III] $\lambda 6312$ and/or [N II] $\lambda 5755$ and determined the $T_{\mathrm{e}}$-based abundances in 10 faint $\mathrm{H}$ II regions in the spiral galaxy M 51. Unfortunately they were unable to detect the oxygen auroral line $R$. We verify whether their faint $\mathrm{H}$ II regions follow the ff-relation in the following way. Using the electron temperature $t_{3}$ recommended by Bresolin et al. (2004) and equations of the $T_{\mathrm{e}}$-method, we have estimated the value of $R$ which corresponds to the $t_{3}$ for every H II region. The bottom panel in Fig. 1 shows the $R$ versus $R_{23}$ diagram for the sample of faint H II regions from Bresolin et al. (2004) (the open squares). The solid line is the ff-relation from the top panel. The positions of faint $\mathrm{H}$ II regions in the $R-R_{23}$ diagram show some kind of bifurcation. It is seen that the oxygen line fluxes in six faint $\mathrm{H}$ II regions (CCM 54, CCM 55, CCM 57, CCM 57A, CCM 71A, and $\mathrm{CCM} 84 \mathrm{~A}$ ) are in good agreement with the ff-relation while the remaining four (CCM 10, CCM 53, CCM 72, and P 203) show significant deviations. The positions of those discrepant $\mathrm{H}$ II regions in the $t_{\mathrm{NII}}$ versus $\mathrm{t}_{\mathrm{SIII}}$ diagram of Bresolin et al. (2004) (their Fig. 2) suggest that their electron temperature determinations should be accurate, and, consequently, that their values of $R$ estimated from the electron temperatures should be reliable. The deviation from the ff-relation is thus probably due to uncertainties in the nebular oxygen line fluxes, and especially in the $R_{2}$ line flux since it makes a dominant contribution to the $R_{23}$ value, the flux in the $R_{3}$ line being small. If this is the case, then the measured $R_{2}$ fluxes in the four H II regions CCM 10, CCM 53, CCM 72, and P 203 are overestimated by 20 to $30 \%$, higher than the errors of $\sim 10 \%$ given by Bresolin et al. (2004). As it was noted above the deviations from the ff-relation can occur if the line fluxes measured within the slit do not reflect their relative contributions to the radiation of the nebula as a whole and this effect would be more important for extended $\mathrm{H}$ II regions. It is interesting to note that at least three of the $\mathrm{H}$ II regions with large $\Delta(\log R)(\mathrm{CCM} 10, \mathrm{CCM} 72$, and $\mathrm{P}$ 203) have significantly larger angular diameters than the H II regions with small $\Delta(\log R)$ in the sample. Bresolin et al. (2004) have noted that large differences were found for CCM 10 and CCM 53 when they compare their line intensities with those reported by other authors.

The residuals $\Delta(\log R)$ from the ff-relation are shown in Fig. 2 as a function of oxygen abundance (top panel) and of excitation parameter $P$ (bottom panel). (H II regions with deviations greater than 0.2 dex were excluded from consideration.) The filled circles are points used for the determination of the ffrelation, the lines are best fits to those data. The open circles are other H II regions with measured $R$. The open squares are those with estimated $R$ from the sample of Bresolin et al. (2004). Figure 2 shows that the differences $\Delta(\log R)$ do not show an appreciable correlation with metallicity or with the excitation parameter for the sample of objects with high-precision flux mesurements.

Thus there seems to be no "third" parameter in the ffrelation, and the scatter in the $R-R_{23}$ diagram seems to be caused either by errors in the flux measurements or by the fact that the measured fluxes do not reflect their relative contributions to the radiation of the whole nebula. We need more highprecision data to confirm or reject this suggestion.

\section{Conclusions}

A relationship between observed auroral and nebular oxygen line fluxes in spectra of H II regions was considered. It was found that this relationship is metallicity-dependent at low metallicities and becomes independent of metallicity (within the uncertainties of the available data) at metallicities higher than $12+\log \mathrm{O} / \mathrm{H} \sim 8.25$, i.e. there is one-to-one correspondence (the ff-relation) between auroral $R$ and nebular oxygen line $R_{23}$ fluxes in spectra of high-metallicity $\mathrm{H}$ II regions over more than three orders of magnitude in $R$.

The ff-relation allows one to estimate the auroral line flux from the measured nebular line fluxes. This solves the problem of the electron temperature (and, consequently, abundance) determination in high-metallicity $\mathrm{H}$ II regions.

The ff-relation confirms the basic idea of the "empirical" method, proposed by Pagel et al. (1979) a quarter of a century ago, that the oxygen abundance in $\mathrm{H}$ II region can be esimated from strong oxygen line measurements only.

Among other things, the ff-relation provides a possibility to select the $\mathrm{H}$ II regions with high quality line measurements and in which measured fluxes reflect their relative contributions to the radiation of the whole nebula. 
A detailed discussion of the application of the ff-relation to abundance determinations in $\mathrm{H}$ II regions will be given elsewhere.

Acknowledgements. It is a pleasure to thank the referee, M. Peimbert, for helpful comments and suggestions. I am grateful to B. E. J. Pagel for constructive comments and suggestions as well as improving the English text. I thank N. G. Guseva, Y. I. Izotov, T. X. Thuan, and J. M. Vílchez for useful discussions. This study was partly supported by grant No 02.07/00132 from the Ukrainian Fund of Fundamental Investigations.

\section{References}

Bressolin, F., Garnett, D. R., \& Kennicutt, R. C. 2004, ApJ, 615, 228

Caplan, J., Deharveng, L., Peña, M., Costero, R., \& Blondel, C. 2000, MNRAS, 311, 317

Esteban, C., García-Rojas, J., Peimbert, M., et al. 2005, ApJ, 618, L95

Esteban, C., Peimbert, M., García-Rojas, J., et al. 2004, MNRAS, 355, 229

Fricke, K. J., Izotov, Y. I., Papaderos, P., Guseva, N. G., \& Thuan, T. X. 2001, AJ, 121, 169

García-Rojas, J., Esteban, C., Peimbert, M., et al. 2004, ApJS, 153, 501

Guseva, N. G., Izotov, Y. I., Papaderos, P., et al. 2001, A\&A, 378, 756

Guseva, N. G., Papaderos, P., Izotov, Y. I., et al. 2003a, A\&A, 407, 75

Guseva, N. G., Papaderos, P., Izotov, Y. I., et al. 2003b, A\&A, 407, 91
Izotov, Y. I., \& Thuan, T. X. 1998, ApJ, 500, 188

Izotov, Y. I., \& Thuan, T. X. 2004, ApJ, 602, 200

Izotov, Y. I., Thuan, T. X., \& Lipovetsky, V. A. 1994, ApJ, 435, 647

Izotov, Y. I., Thuan, T. X., \& Lipovetsky, V. A. 1997, ApJS, 108, 1

Izotov, Y. I., Chaffee, F. H., \& Green, R. F. 2001, ApJ, 562, 727

Izotov, Y. I., Papaderos, P., Guseva, N. G., Fricke, K. J., \& Thuan, T.X. 2004, A\&A, 421, 539

Kennicutt, R. C., Bresolin, F., \& Garnett, D. R. 2003, ApJ, 591, 801

Lee, H., McCall, M. L., Kingsburgh, R. L., Ross, R., \& Stevenson, C. C. $2003 \mathrm{a}, \mathrm{AJ}, 125,146$

Noeske, K. G., Guseva, N. G., Fricke, K. J., et al. 2000, A\&A, 361, 33

Oey, M. S., Dopita, M. A., Shields, J. C., \& Smith, R. S. 2000, ApJS, 128,511

Oey, M. S., \& Shields, J. C. 2000, ApJ, 539, 687

Pagel, B. E. J., Edmunds, M. G., Blackwell, D. E., Chun, M. S., \& Smith, G. 1979, MNRAS, 189, 95

Peimbert, A. 2003, ApJ, 584, 735

Pilyugin, L. S. 2000, A\&A, 362, 325

Pilyugin, L. S. 2001a, A\&A, 369, 594

Pilyugin, L. S. 2001b, A\&A, 373, 56

Pilyugin, L. S. 2003, A\&A, 399, 1003

Thuan, T. X., Izotov, Y. I., \& Foltz, C. B. 1999, ApJ, 525, 105

Thuan, T. X., Izotov, Y. I., \& Lipovetsky, V. A. 1995, ApJ, 445, 108

Tsamis, Y. G., Barlow, M. J., Liu, X.-W., Danziger, I. J., \& Storey, P. J. 2003, MNRAS, 338, 687

Vermeij, R., Damour, F., van der Hulst, J. M., \& Baluteau, J.-P. 2002, A\&A, 390, 649

Vílchez, J. M., \& Iglesias-Páramo, J. 2003, ApJS, 145, 225 